\title{
Teoria para la construcción de una ciudad: Enrico Tedeschi y su vínculo con la morfología urbano-edilicia de Mendoza (Argentina, 1960)
}

\section{Cecilia Raffa}

(Mendoza, 1975). Es arquitecta (Universidad de Mendoza), magíster en Arte Latinoamericano y doctora en Ciencias Sociales (Universidad Nacional de Cuyo). Se desempeña como investigadora del Instituto de Ciencias Humanas, Sociales y Ambientales del Consejo Nacional de Investigaciones Científicas y Técnicas (INCIHUSA- CONICET, Argentina). Su especialidad es la historia cultural de la arquitectura y la ciudad del siglo XX, desde donde aborda estudios sobre la arquitectura pública y las relaciones entre el campo técnico y el campo político; desarrolla además trabajos en torno del Patrimonio Cultural. Fue directora e integrante del equipo responsable de diversos proyectos de investigación científica financiados por organismos provinciales y nacionales (FPC, FNA, CONICET, ANPCyT, etc.). Codirige becas doctorales y posdoctorales del CONICET y es docente de grado y posgrado. Escribió numerosos trabajos publicados en libros y en revistas científicas nacionales e internacionales. Es autora del libro Plazas fundacionales. El espacio público mendocino entre la técnica y la política, Mendoza 1910-1943 y compiladora y coautora de Profesionalizando un Estado provincial. Mendoza 1890-1955 y Arquitectos en Mendoza, ambos editados por la UNCuyo. craffa@mendoza-conicet.gob.ar

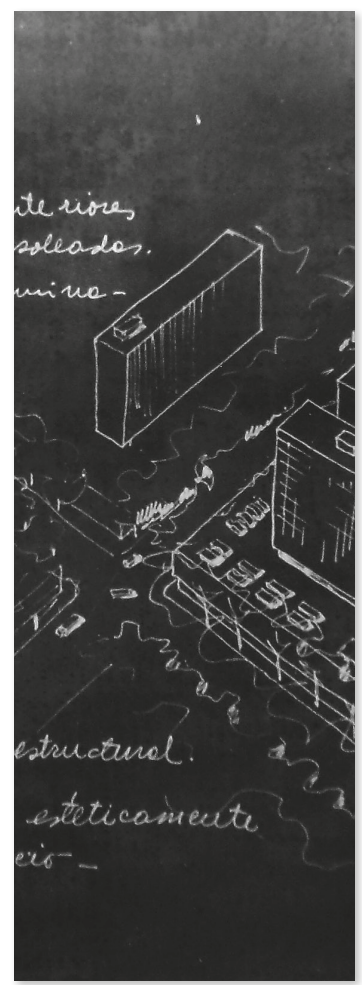




\title{
Resumen
}

ENRICO TEDESCHI es una figura clave en el panorama de la modernidad en Mendoza. Instalado desde 1948 en Argentina, su actuación profesional estuvo signada por la dedicación a la enseñanza y a la investigación de la Historia y la Teoría de la Arquitectura. Pero dentro de su trayectoria profesional fueron también importantes sus aportes en el campo del urbanismo: TEDEschi tuvo un papel preponderante en la Comisión Especial de Planeamiento Urbano y Código de Edificación de la ciudad de Mendoza.

Nuestro abordaje hipotetiza sobre la relación entre la producción teórica de TEDESCHI plasmada en su libro Teoría de la Arquitectura y el informe que elabora para la Comisión de Planeamiento y Código de Edificación. Supone además que el primer informe tuvo injerencia en la morfología urbano-edilicia reglamentada en el Código de Edificación de Mendoza, que afectó a las planificaciones urbanas de toda la provincia.

\section{Palabras clave}

Planificación urbana; Enrico Tedeschi; Mendoza; trayectorias profesionales; Estado.

\begin{abstract}
s
A theory for the building of a city: Enrico Tedeschi and his links to the urban - architectural morphology of the city of Mendoza (Argentina, 1960).

Enrico Tedeschi was a key figure in the panorama of modernity in Mendoza. Since his arrival in Argentina in 1948, his professional activities were marked by his dedication to teaching and researching the History and Theory of Architecture. But within his career, his contributions in the field of urbanism were also important as he played a leading role on the Special Commission on Urban Planning and Building Code of the city of Mendoza. Our approach considers the relationship between his theoretical production embodied in his book Theory of Architecture and the report he produced for the Planning and Building Code Commission. It also assumes that the his first report had an influence on the urban-architectural morphology as regulated in the Building Code of Mendoza, which resulted in influencing urban planning throughout the province.
\end{abstract}

\section{Keywords}

Urban planning; Enrico Tedeschi; Mendoza; Professional activities. 


\section{Enrico Tedeschi ${ }^{1}$}

Entre 1945 y 1946, EnRico Tedeschi ejerció el cargo de jefe de la oficina de Urbanismo, en la Subsecretaría de Bellas Artes del Ministerio de Instrucción Pública de Italia. Fue el cargo público más importante desempeñado por este arquitecto romano antes de emprender su viaje a América, y que de alguna manera tuvo su correspondencia en Argentina, al asumir la jefatura de la oficina técnica de la Comisión Especial de Planeamiento Urbano y Código de Edificación de la ciudad de Mendoza en $1960 .^{2}$

Desde su graduación como arquitecto en 1934, TEDEschi comenzó un camino que gravitaría entre el ejercicio profesional, desarrollado fuertemente en Europa, y la docencia y la investigación, actividades que tuvieron en América su lugar de expansión. En su extenso currículum, se destaca su temprano interés por el urbanismo como especialidad, vocación interrumpida por su participación en la guerra en el norte de África entre 1940 y 1943, pero que siguió desplegando luego, nuevamente en Italia y en Argentina. El concurso nacional para el Plano Urbanístico de Aprilia (1935), la realización del Plano Urbanístico de la zona del Circeo (1940), la revisión del Plano Regulador de la ciudad de Roma (1944-1945), la confección del Plan Regulador para las ciudades de Caserta (1945-1946) y Capua (1946-1947), entre otros, junto a publicaciones referidas a temas urbanos (como el boletín de reconstrucción urbanística solicitada por el Servicio Cultural de EE. UU., en 1946), el dictado de clases de urbanismo en Roma (1946) y Venecia (1948) y su participación como vocal del Instituto Nacional de Urbanismo de Roma entre 1946 y $1947^{3}$ marcan el interés de TEDEschi en torno a la planificación de las ciudades. Estas experiencias sumadas a su papel como proyectista en Italia fueron perfilando su concepción de la arquitectura y la ciudad con un amplio sentido cultural de base histórica-crítica y orgánica, visión que quedó expresada en sus libros y que pudo plasmar en los proyectos de los que participó en Argentina, principalmente en las ciudades de Tucumán y Mendoza.

De su estancia en el norte argentino entre 1948 y 1953, podemos destacar la confección del Plano Urbanístico de la Ciudad Universitaria de Tucumán, realizado en colaboración con otros profesionales bajo la dirección del arquitecto Jorge Vivanco, y la publicación junto a Cino Calcaprina del escrito Urbanismo con Legislación, editado por la Universidad Nacional de Tucumán en 1950. La Facultad de Arquitectura de la Universidad Nacional de Córdoba lo tuvo como profesor en el período 1953-1959. Allí tuvo una participación destacada en la
1. Este artículo está basado en la ponencia: "Enrico Tedeschi. Aportes teóricos en la construcción de ciudad (Mendoza-Argentina, 1960)", presentada en el Primer Congreso Iberoamericano de Historia Urbana, realizado en Santiago de Chile en 2016.

2. Trabajos recientes revisan el derrotero de Tedeschi (Roma, 1911; Buenos Aires, 1978) en Argentina. Algunos ponen el énfasis en sus aportes sobre teoría, historia y educación en la Arquitectura: SELLA Y ADAGIO (2013); MALECKI (2013: 137174) Y ADAGIO ET ÁL. (2017); a su producción de arquitectura y su relación con el paisaje: ALVITE (2015: 73-86; 2013 : 476-484) y a sus redes de relaciones profesionales: CIRVINI Y RAFFA (2014: 86-101).

3. Enrico Tedeschi, Legajo FAU-UM. 
4. Aparece en su $\mathrm{CV}$ la realización de una investigación urbanística sobre Mendoza realizada en 1955; es probable que se trate de los estudios que lleva delante desde la División Mendoza de la Sociedad Central de Arquitectos. formación del Instituto Interuniversitario de Historia de la Arquitectura y formó parte de la Comisión de Estudios de la Ciudad Universitaria entre 1957 y 1960.

En 1954, y mientras se desempeñaba como profesor también en la Universidad Nacional de Cuyo sede San Juan, TEDEsCH formó parte del grupo de profesionales que organizó la División Mendoza de la Sociedad Central de Arquitectos, institución a través de la cual se acercó a la problemática urbana de Mendoza participando en diversas comisiones internas que trataron temas como la ley de loteos y el código de edificación, ${ }^{4}$ y que se ocuparon de la edición de una recopilación de ordenanzas municipales sobre construcción (División MendozA, 1953-1959). Fueron esos ámbitos los que afianzaron las relaciones personales y profesionales que lo vincularon, entre otros, con Daniel Ramos Correas y Raúl Panelo Gelly, integrantes luego de la Comisión Especial de Planeamiento Urbano y Código de Edificación de Mendoza (en adelante, CEPyCE) y co-organizadores de la Facultad de Arquitectura de la Universidad de Mendoza (1961), de la que Tedeschi fue decano fundador (Cirvini y RAFFA, 2014; RafFA, 2015).

Entendemos que existió una directa relación entre la propuesta metodológica de TEDESCHI plasmada en su libro Teoría de la Arquitectura y las bases del concurso para técnico urbanista, cuyos requerimientos integraron el informe elaborado para la CEPyCE. Consideramos, además, que de las formulaciones incluidas en el primer informe tuvieron injerencia real aquellas relacionadas con el desarrollo de la morfología urbano-edilicia reglamentada en el Código de Edificación de Mendoza. Propuestas y relaciones que quedarán enunciadas en este artículo, sobre las cuales seguimos investigando.

Sobre la base del método comparativo, y partiendo de un contexto general sobre los debates en torno al urbanismo en Argentina a mediados del siglo XX, trabajaremos analizando cuatro fuentes principales: los contenidos del primer informe de la oficina de planeamiento dirigida por TeDEschi; la primera edición del Código de Edificación de Mendoza, el libro Teoría de la Arquitectura y finalmente la publicación que sintetiza la labor de la Comisión de Planeamiento y Código de Edificación de Mendoza.

\section{Urbanismo en Argentina (1940-1960c)}

Cuando Tedeschi se instaló en Tucumán en 1948, los problemas de ornato y de equipamiento urbano (aperturas, ensanches, extensiones y embellecimiento), que eran centrales para los 
paisajistas, higienistas, ingenieros y más tarde arquitectos a principios de siglo, se habían desplazado para dar lugar a la preocupación por el alojamiento de masas, el zooning y la circulación. Los planes reguladores formulados para varias ciudades argentinas, la larga reconstrucción de San Juan después del terremoto de 1944, incluso las políticas masivas de vivienda del gobierno peronista $^{5}$ (1946-1955) que impactaron fuertemente en el Gran Buenos Aires y en las áreas centrales de varias de las principales provincias fueron imprimiendo características particulares a las problemáticas urbanas en el país (Novick y Piccioni, 2004).

Por un lado, los planes reguladores prometieron encauzar el desarrollo y la integración social y económica de las ciudades y el territorio desde una perspectiva tecnocrática (RigotTi, 2009). Se imaginaron ciudades como proyectos a gran escala, con áreas de usos definidos, que recuperarían la armonía alguna vez existente entre hombres, naturaleza y objetos. Organizados a partir de "expedientes urbanos" cuyo objetivo era radiografiar las particularidades de la ciudad y sus males, el grado de intervención propuesta superó el de algunos conjuntos edilicios o ejes viales de las primeras décadas del siglo XX, para alcanzar la totalidad de la planta urbana y sus posibles extensiones en el tiempo y el espacio.

Esos planes, cuyo marco de referencia se desplazó desde Francia a EE. UU. ${ }^{7}$ y entre los que están el de Mendoza $(1940)^{8}$ y el plan ideado para

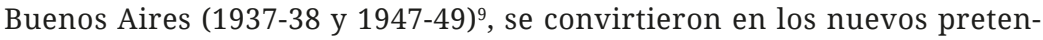
didos instrumentos de gestión urbana. Con escasas concreciones a raíz de la confluencia de factores políticos, económicos y hasta disciplinares, las propuestas se pensaron como imágenes, con fuerte carga formal, capaces de guiar la acción colectiva y compleja que construía las ciudades (RigotTi, 2014).

La experiencia de la reconstrucción de San Juan, por su parte, no llegó a constituirse - pese a sus potencialidades - en un laboratorio de urbanismo. La organización de una nueva ciudad moderna se fue diluyendo, en la medida en que las promesas de ejecución de los distintos planes oficiales que

\section{Al respecto, ver entre otros BALLENT (2009).}

6. En 1919, la Société Française des Urbanistes determinó las bases de los planes reguladores, integradas por expediente urbano, programa, anteproyecto, reglamento y memoria (RIGotTI, 2014: 191). Este formato fue utilizado como modelo para la disciplina.

\section{Circulaban en el momento de la} elaboración de estos planes las experiencias norteamericanas del New Deal, tanto como las planificaciones de posguerra europeas.

8. El Plan Regulador para Mendoza de 1940 fue confeccionado por el equipo de Fermín Bereterbide, Alberto Belgrano Blanco, Mauricio Cravotto y Juan Scasso, quienes ganaron un concurso organizado por el Estado provincial. Tuvo como objetivo buscar soluciones para regular la edilicia y la distribución de infraestructuras y servicios, la extensión desordenada de la planta urbana, la insalubridad y el desorden funcional (RAFFA, 2014).

9. El plan que comenzó a bocetar $L E$ CORBUSIER en 1929 en su visita a la ciudad fue elaborado por Jorge Ferrari Hardoy y Juan Kurchanen París entre 1937 y 1938, y se disolvió en el fallido intento de implementación por parte del gobierno de la ciudad, entre 1947 y 1949 (LIERNUR y PSCHEPIURCA, 2008). En París nacería el germen del grupo Austral, fundado en 1938 en Buenos Aires. 
10. Nos referimos a los planes realizados por los arquitectos y urbanistas Fermín Bereterbide y Ernesto Vautier (1944); Ángel Guido-Benito Carrasco (realizado dos años antes del terremoto por encargo del gobierno provincial y que se pretendió reactivar luego del terremoto); Jorge Ferrari Hardoy, Jorge Vivanco, Simón Ungar, Alberto Le Pera y Samuel Oliver (1944), Julio Villalobos (1945), Carlos Mendióroz (1945). Para un análisis detallado, ver HEALY (2012) y RIGOTTI (2014).

11. Ya estaban definidas las características generales para la reedificación, siguiendo las propuestas del plan de Mendióroz (HEALY:

2012, 280).

12. Pastor tomó a su cargo un plan en ejecución de líneas moderadamente modernas y trabajó sobre la unificación y coordinación de los accesos ferroviarios y viales y la fijación de criterios para regular y reformar las áreas edificadas (HEALY: 2012, 280 y ss.). Sobre la trayectoria de Pastor, ver RIGotTI (2014).

13. Sobre las particularidades de la instalación de estos arquitectos italianos en el país y la historia de los debates en torno del urbanismo en Argentina, recomendamos RIgotTI, 2014.

14. Los últimos proyectos mencionados, si bien fueron ideados cuando el instituto había desaparecido (en 1952 cambió su nombre por Facultad de Arquitectura y Urbanismo), manifiestan la influencia directa de los postulados internacionales (CIRVINI Y RAFFA, 2014). se sucedían y no se ejecutaban fueron perdiendo credibilidad. ${ }^{10}$ La reconstrucción de la ciudad se puso en marcha en 1948 dirigida por José M. Pastor, sin llegar a concretarse ${ }^{11}$ Con fuertes influencias de las operaciones de Abercrombie y Forshaw para Londres (RigotTi 2014), pero poniendo atención en las particularidades del caso, Pastor persiguió la planificación de una ciudad y sus edificios de manera integral, siguiendo los principios modernistas generales $^{12} \mathrm{y}$ desplazando el interés por la dimensión física a la sociedad humana, como constructora de las ciudades. En ese contexto de proyecciones de futuro para distintas ciudades que no habían podido materializarse del todo, de distintas escalas de actuación y de diversos marcos teóricos, TEDEsCH formó parte del grupo de italianos que influyeron en las prácticas convencionales de la disciplina urbanística en el país. ${ }^{13}$

Junto a Cino Calcaprina y Luiggi Piccinato, TeDeschi participó en la formulación de planes y proyectos para el norte argentino, entre los que están el proyecto para la Ciudad Universitaria de Tucumán, la Ciudad Hospital en Horco Molle, viviendas obreras para los ingenios Marapa y Nuñorco, escuela y hotel en Purmamarca, Centro Cívico en Catamarca y el Plan Regulador de Jujuy-Palpalá ${ }^{14}$. A un ambiente fuertemente "actualizado", tal como sostiene RigotTi, por las posiciones peninsulares y norteamericanas (Le Corbusier, Sert, Tennessee Valley Authority, Regional Planning Association of America), y marcado por figuras como Horacio Caminos y Jorge Vivanco (allegados al grupo Austral), los italianos contrapusieron una alternativa de discusión "orgánica” que ponía en relación modernidad y tradición (CiRvini y RAFFA, 2014). Esta opción de la arquitectura y el urbanismo orgánicos posicionaba el "espacio" sobre la "plástica", volviéndolo a potenciar, tal como había ocurrido en otros momentos de la historia de la arquitectura (Tedeschi, 1951). Se presentaba realista, a escala humana y respetuosa de los datos de la naturaleza.

Fue a partir de la creación del Instituto de Arquitectura y Urbanismo, en el que confluyeron posiciones diversas (perspectiva corbuseriana y organicismo italiano, principalmente), que Tucumán se convirtió en un semillero de experiencias, producto de un contexto histórico, que la generación a la cual perteneció TEDESCHI trasladó a distintos puntos del país (CIRvinI y RAFFA, 2014). 
Mendoza fue uno de esos destinos. Fue allí donde TEDEschi encarnó el papel del arquitecto urbanista: el planificador. Sobre la base de una formación histórico-crítica, de su experiencia europea y del estudio de los valores del paisaje local, pudo coordinar a un grupo de especialistas para desarrollar diagnósticos y propuestas parciales, que servirían como guía a la relegada planificación de la ciudad de Mendoza.

\section{Tedeschi en la Comisión Especial de Planeamiento Urbano y Código de Edificación ${ }^{15}$}

La CEPyCE fue creada por la Ordenanza Municipal 1729 del año 1959, y se formó luego de una serie de frustrados intentos de planificación de la ciudad que se venían sucediendo desde 1915 con el "Proyecto de Ampliación y Rectificación del trazado para la ciudad de Mendoza” de Benito Carrasco, a través de numerosas ordenanzas y leyes de escala provincial y municipal, hasta llegar al concurso para el "Plan Regulador de la Ciudad de Mendoza" en los años $40 .{ }^{16} \mathrm{El}$ objetivo fundamental de la organización de este grupo fue analizar y detectar los procedimientos para hacer plausible el "Planeamiento Urbano y el Código de Edificación de la Ciudad de Mendoza”, teniendo en cuenta que esa ciudad formaba parte de lo que se denominaba el "Gran Mendoza". ${ }^{17}$

La comisión que asumía la supervisión y dirección de los trabajos estuvo compuesta por tres representantes del Honorable Concejo Deliberante, el secretario Municipal de Obras Públicas e Higiene, el director Municipal de Obras Públicas y el asesor letrado de la comuna y una serie de delegados de distintas instituciones íntimamente relacionadas con la construcción de la ciudad: el arquitecto Raúl Panelo Gelly y el ingeniero Salomón Darwich por Consejo Profesional de Ingenieros, Arquitectos y Constructores; Pedro Zuppo de la Sociedad de Constructores de Obras y anexos; el ingeniero Justo Pedro Gascón del Centro de Ingenieros, Arquitectos y Agrimensores y Daniel Ramos Correas por la Sociedad de Arquitectos de Mendoza. ${ }^{18}$

Los integrantes de la comisión promovieron encuentros con los intendentes de los departamentos circunvecinos de Mendoza, con el objetivo de mancomunar
15. Mediante Resolución 2258/1958, se creó en el ámbito municipal la Comisión Especial procódigo de edificación, que no tuvo una labor destacada y fue reemplazada un año después por la CEPYCE conservando a sus integrantes (PANELO GeLLY, 1961: 25-26).

16. Son escasas las investigaciones que abordan el estudio de los aspectos urbanos de Mendoza en clave histórica. Entre los clásicos están PONTE (2008) y BÓRMIDA (1984) y más recientemente indagaciones que trabajan entre 1910 y 1940 (RAFFA, 2016), o sobre planes específicos (RigotTi, 2014). Por consiguiente este artículo supone un avance en el estudio de las propuestas urbanas para la ciudad de Mendoza, durante la segunda mitad del siglo $X X$.

\section{El Gran Mendoza (hoy Área} Metropolitana) estaba constituido por los departamentos de Luján, Las Heras, Maipú, Godoy Cruz, Guaymallén y Mendoza.

18. En la ordenanza de conformación de la comisión se designa a Daniel Ramos Correas como su presidente. Si bien el desempeño de los miembros de la comisión no fue rentado, se contó con una partida de un millón de pesos anuales entre 1960 y 1961 para el cumplimiento de los objetivos (PANELO GELLY, 1961: 31). 
19. En junio de 1961, a instancias de la CEPyCE, se acordó con representantes municipales y provinciales un proyecto de convenio que finalmente nunca se concretó, razón por la cual el estudio se limitó a la ciudad de Mendoza (PANELO GELLY, 1961: 32-33).

20. El concurso para especialista en estructuras fue adjudicado al ingeniero

Francisco Giuliani, en noviembre de 1960 (PANELO GELLY, 1961: 32-33). fuerzas en la resolución de los problemas urbanísticos que, por la conformación urbana del Gran Mendoza, le competían y afectaban a todas las jurisdicciones municipales. A la reunión celebrada en noviembre de 1959 asistió ENRICO TEDESCH invitado por la comisión, como especialista en urbanismo: fue el único convocado para estas reuniones con los municipios. Un mes después, la CEPyCE decidió la contratación de un profesional urbanista por concurso, que realizara el estudio de los antecedentes necesarios para hacer posible el planeamiento de la ciudad capital, con vistas al Gran Mendoza, y de un profesional especializado en estructuras de edificios, con el objetivo de elaborar el Código de Edificación. ${ }^{19}$ Los concursos se abrieron en julio de 1960. Las bases fueron preparadas por los arquitectos Ramos Correas y Panelo Gelly, y por el ingeniero Darwich para el cargo de urbanista, y por el ingeniero Justo Pedro Gascón para el del especialista en estructuras. ${ }^{20}$ Ambas tuvieron difusión nacional.

El concurso para la jefatura de la Oficina de Planeamiento se orientó a profesionales urbanistas radicados en Argentina, de cualquier nacionalidad o título, quienes debían exponer su experiencia en la temática. El profesional seleccionado debía conocer el medio, la región y sus problemas, y poseer suficiente prestigio y personalidad como para cooperar positivamente en la conformación de la conciencia urbanística de sus colaboradores y de la población en general. El llamado supuso la contratación de un urbanista capaz de organizar y dirigir los estudios previos para obtener la documentación que hiciera posible el planeamiento urbano de la ciudad de Mendoza y la redacción de su Código de Edificación. La labor debía cumplirse en dos etapas: en la primera el técnico entregaría a los seis meses de iniciado su contrato un informe en el que indicase las medidas urgentes que tomar por el Municipio en tanto se concluía el estudio general; en la segunda, fijada a los seis meses de la primera, debía aportar la documentación completa de datos y antecedentes para la confección del plan, que incorporaría un análisis sobre el paisaje natural, la estructura urbana, el paisaje cultural (incluyendo relaciones con la región), el uso de la región, demografía general, aspectos demográficos socioeconómicos, el estado de la edificación, de las normas sobre edificación, de los servicios públicos, de los servicios sociales y culturales, de la vialidad, el transporte, las actividades industriales, comerciales, turísticas y administrativas (Panelo Gelly, 1961).

El técnico designado estaría al mando de un equipo aportado por la Municipalidad de la capital y la Dirección de Arquitectura de la provincia, cuyos integrantes fueron el arquitecto 
Simón Lacerna como secretario técnico, los arquitectos Martín Abraham y Pedro Merlo como investigadores, ${ }^{21}$ Armando Gei, Ángel Meza, Juan Carlos Mucha y Andrés Bernhard como dibujantes; el arquitecto Raúl Panelo Gelly como secretario relator y un grupo de administrativas y ordenanzas (PANelo Gelly, 1961). La elección de TeDEschi para ocupar el cargo fue unánime. En noviembre de 1960 se firmó el contrato entre la comuna capitalina y el urbanista.

El perfil profesional de TEDEschi coincidía claramente con el pedido en el concurso, tanto por sus antecedentes como por su conocimiento sobre la región. Pero además, la particular demanda dentro de las bases de los dieciséis aspectos básicos que definir por el técnico urbanista, muy cercanos a los postulados elaborados por Tedeschi en su libro Teoría de la Arquitectura publicado en 1962, nos hacen suponer que existió una fuerte vinculación en la antesala del concurso, basada en las redes de relaciones a las que nos hemos referido, entre los agentes de la CEPyCE y TEDESCHI, que resultó en la contratación de este último.

En efecto, la metodología propuesta por TeDEschi en Teoría de la Arquitectura, ${ }^{22}$ como base para la formulación de un programa arquitectónico, resulta aplicable en su primera parte a un estudio urbanístico, y entendemos que fue utilizada antes de su publicación final, ya que una versión inicial había sido editada en 1957 en Córdoba, ${ }^{23}$ para analizar la ciudad de Mendoza. Particularmente nos referimos al estudio de los paisajes natural — terreno, clima y vegetación-y cultural: la región, la ciudad como hecho físico, social y estético, la forma, y de la demografía, concurrentes en ambas propuestas. Junto a esos aspectos, podemos indicar que el abordaje de las actividades, el transporte y los servicios enunciados en las bases del concurso son comparables al estudio de los valores político-administrativos y económicos de producción y de intercambio propuestos por TEDEschi en su libro para el análisis del paisaje cultural urbano.

El tema del paisaje había aparecido ya como preocupación de TEDEschi en La Plaza de Cuzco, escrito en 1949 y editado en 1961, en el que proponía que el elemento natural debe ser pensado en equivalencia con el arquitectónico (Alvite, 2015). Está contenido también en el capítulo "Espacio Externo. Urbanismo y Paisaje” de Una introducción a la Historia de la Arquitectura (1951) y explicitado en un artículo publicado en Nuestra Arquitectura en 1954 (TEDESCHI, 1954), en que sostenía que era necesario que todo urbanista considerara también los factores estéticos del plan (en el caso de Mendoza, las consideraciones sobre la edificación
21. Sobre las trayectorias profesionales de Lacerna, Merlo, Abraham y Ramos Correas, recomendamos ver CECILIA RAFFA (2017).

22. Nos referimos a los esquemas incluidos en ENRICO TEDESCHI (1962: 285 y ss).

23. Esta publicación de 1957 supone cierta circulación del texto en el campo técnico y académico (MALECKI, 2013: 137-174). 
24. El texto conocido como "informe Tedeschi" se extravió de los archivos municipales; solo se conserva una copia sin los anexos gráficos ni las conclusiones en la biblioteca del Colegio de Arquitectos de Mendoza.

25. Entre las actividades ejecutadas por la oficina, se destaca la organización de las Jornadas de Urbanismo de 1960 y de una serie de tareas de difusión a través de la prensa (OFICINA DE Planeamiento, 1961: 17 y ss.). existente y futura que aparecen en los informes de la oficina de planeamiento), a partir del estudio de los valores del paisaje, basados en una formación histórico-crítica del arquitecto. Esa es la premisa que sugieren las bases del concurso y que siguió TEDEscH en la elaboración de su estudio urbano.

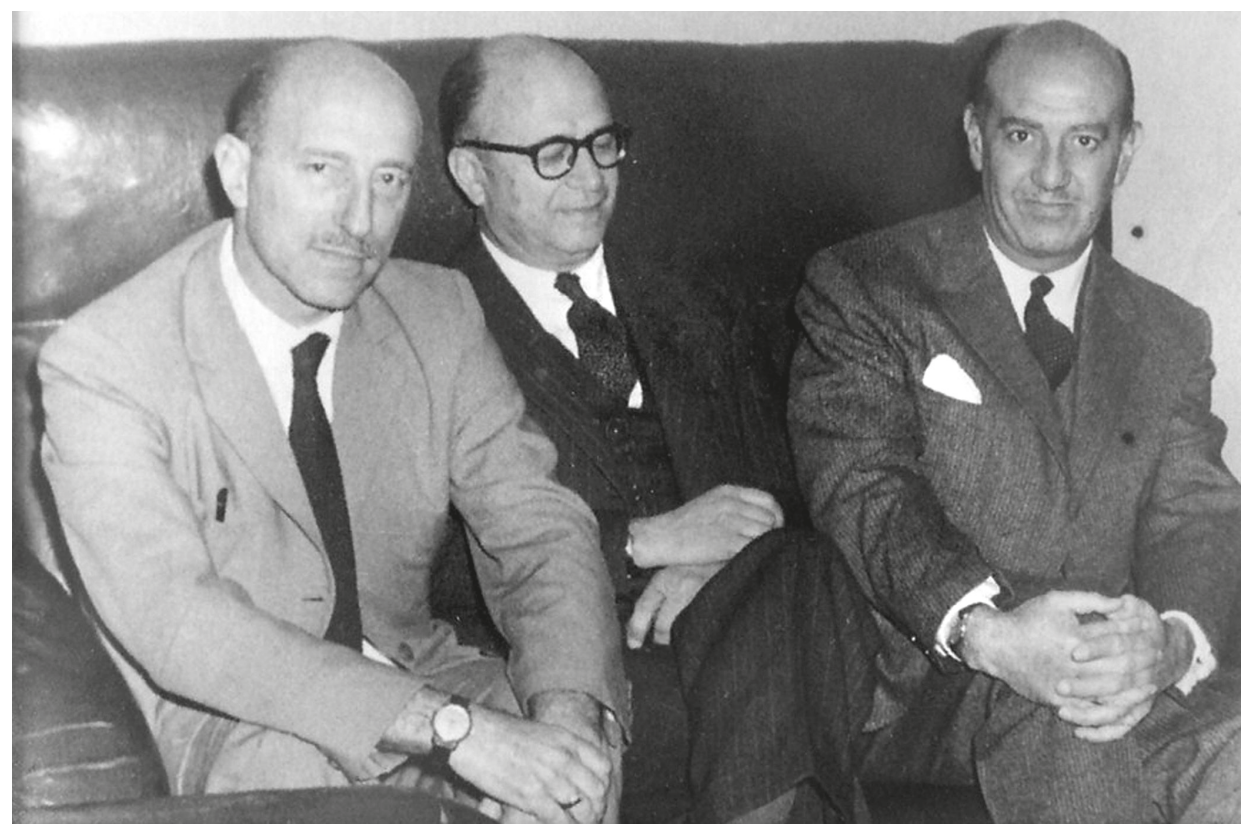

Figura 1. Enrico Tedeschi, Daniel Ramos Correas y Raúl Panelo Gelly, 1960. Fuente: Archivo de la Sociedad de Arquitectos de Mendoza

\section{La teoría y los aportes urbanos: primer informe $e^{24}$}

El primer informe fue presentado en febrero de 1961 por TEDEschi y su equipo. El escrito contenía un resumen de lo actuado por la Oficina de Planeamiento hasta ese momento, ${ }^{25}$ sumado a una serie de medidas y resoluciones que se aconsejaba al Ejecutivo Municipal tomar, 
en tanto se terminaban los estudios de planificación urbana. Para entonces, la oficina se encontraba en pleno proceso de recopilación de documentación útil al análisis del paisaje natural (cartografía, datos referidos al clima, sobre aguas superficiales y perforaciones y tipos de vegetación), utilizando como base la información recopilada para el Plan Regulador de 1940. Del mismo modo, se indicaba el avance de las indagaciones sobre el paisaje cultural a partir de la recopilación de datos en torno a la estructura urbana, la demografía (para los que se utilizaron fichas del Censo Poblacional de 1960), el análisis del estado de la edificación y de la normativa vigente y de la situación administrativa de la comuna.

Si bien la investigación llevada adelante no suponía soluciones efectivas al planeamiento de la ciudad, algunas de las medidas generales sugeridas al Ejecutivo a través de la CEPy$\mathrm{CE}$ avanzaron en disposiciones urbanas y fueron las que, independientemente del estudio completo presentado en el informe final, se tomaron como referencia para el Código de Edificación de 1970. Estas disposiciones están relacionadas principalmente con la regulación de densidades edilicias, para lo cual se recomienda la densificación de la ciudad, comparando Mendoza con otros centros urbanos (Madrid, Roma, Florencia y Córdoba); el establecimiento de un perfil escalonado para los edificios en altura y la protección de la arboleda urbana. Indicaciones todas que poseen una fuerte impronta ambiental explicitada en su preocupación por el asoleamiento, las orientaciones, la circulación de los vientos, etc., característica que irá adquiriendo cada vez más protagonismo en la trayectoria profesional de TEDEschi, hasta convertirlo en uno de los precursores en el desarrollo de la arquitectura solar en Argentina. ${ }^{26}$

Ahora bien, ¿cuál fue la traducción de esas recomendaciones en el Código de Edificación? La principal influencia está en referencia a la edificación en altura en la ciudad y su relación directa con las particulares del paisaje urbano. El informe incluye esquemas que grafican cómo la ciudad y sus habitantes, a partir del incremento de la edificación en altura, estaban perdiendo el acceso al sol y a la ventilación, hecho que iba en detrimento también de la arboleda urbana. Del mismo modo, cuestiona la disposición de construir con alturas mínimas en ciertos sectores de la ciudad, como obstáculo para la renovación edilicia. Como propuesta, incorpora bosquejos de tipos especiales de edificios, adoptados en otros lugares del mundo, como posibles aportes para Mendoza. Esta propuesta junto a la derogación de la obligación de edificar con alturas mínimas en las calles céntricas permitiría el tratamiento de la manzana como unidad orgánica. Así, la recuperación de la amplitud para el ingreso del sol y el aire
26. A partir de 1973,

TEDESCHI se dedicó a la investigación. Fundó el Instituto de Arquitectura y de Urbanismo de la FAUUM; el IADIZA, Instituto Argentino de Investigación de Zonas Áridas, y el LAHV, Laboratorio de Ambiente Humano y Vivienda, hoy INAHE, Instituto de Ambiente, Hábitat y Energía, pertenecientes estos dos últimos Institutos al CONICET (CIRVINI Y RAFFA, 2014). 
Figuras 2, 3, 4, y 5 . Esquemas de edificación en altura: efectos visuales y asoleamiento $y$ efectos acústicos y asoleamiento y arboleda urbana. Fuente: Oficina de Planeamiento (1961) en los canales urbanos se daría a partir de un basamento de hasta cuatro metros de altura, con el desarrollo del resto del edificio desde un retiro frontal de quince metros de la línea municipal y con la determinación de alturas máximas que construir en la zona comercial céntrica. La propuesta suponía que la terraza que se formaba con el retiro sirviera como estacionamiento vehicular, para contrarrestar los problemas que acarreaban el creciente tráfico automotor y la falta de lugares para su aparcamiento (Oficina de Planeamiento, 1961).

Demostrando su perfil histórico-crítico, TEDESCHI incorpora en estos gráficos la comparación entre las construcciones bajas de Mendoza (etapa pos-terremoto) remarcando sus ventajas respecto de las posibilidades de asoleamiento y ventilación; las que se hacían al momento del informe y las edificaciones que deberían hacerse recuperando, con una tipología distinta de la de las antiguas casas, el acceso al sol y al aire y preservando la arboleda urbana.
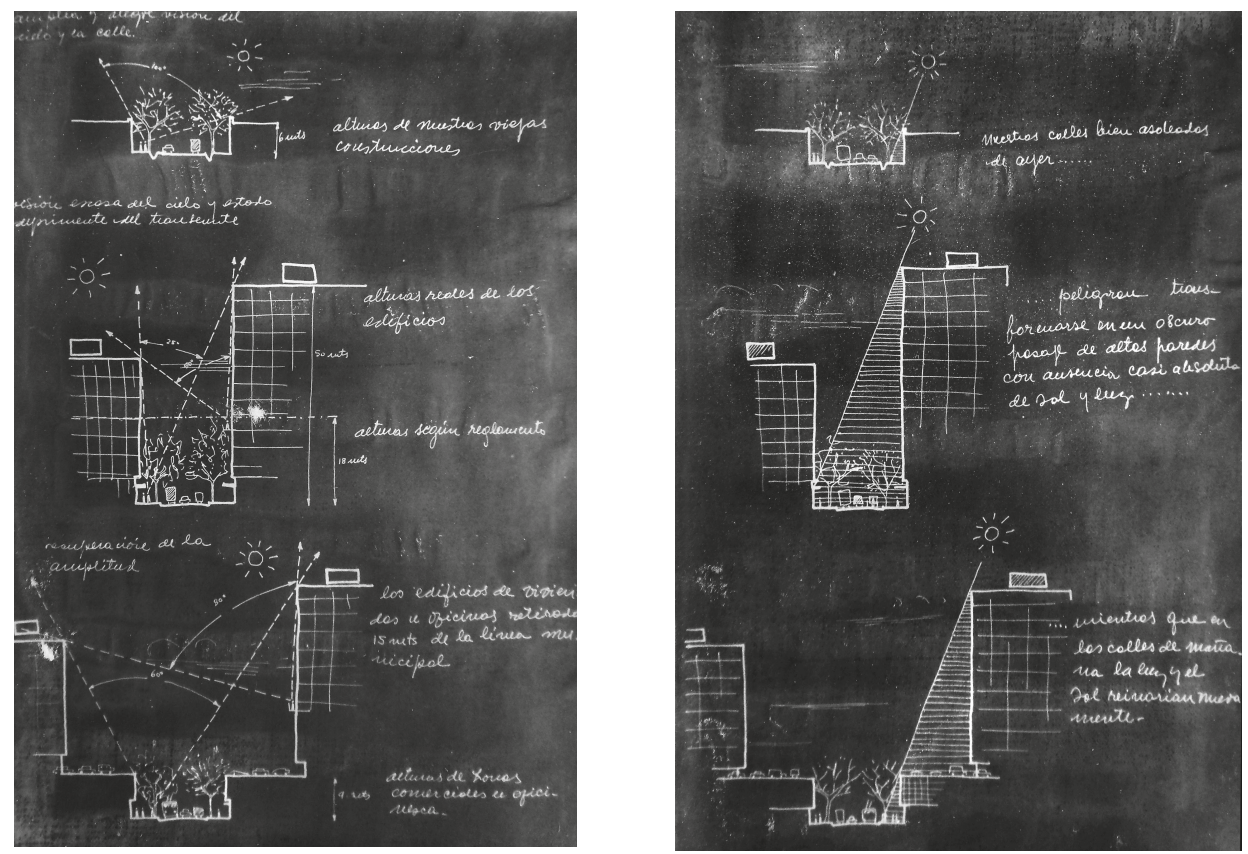
Con ajustes, el Código de Edificación de 1970 incorpora estas recomendaciones introduciendo la obligatoriedad del retiro de los cuatro laterales en edificios de altura con un basamento máximo de diez metros. Ese retiro nunca sería menor a tres metros, y resultaría de la fórmula $\mathrm{R} \geq 1+\mathrm{h} / 10$, donde $\mathrm{R}$ es el retiro mínimo y $\mathrm{h}$ la altura total de la construcción medida desde la cota de vereda. En relación con esta disposición, el código regula las salientes sobre la vía pública determinando que su distancia a troncos o ramas importantes de árboles no sea inferior a $0,40 \mathrm{~m}^{27}$. Para la utilización de los retiros, y probablemente teniendo en cuenta que al dividir el retiro obligatorio en los cuatro laterales del edificio la superficie de cada uno disminuiría (no serían los $15 \mathrm{~m}$ de TEDEschI), se contemplaba su uso como circulación para la instalación de juegos infantiles, confiterías al aire libre y toda otra función que no supusiera una molestia a los vecinos y que no afectara la estética del edificio.
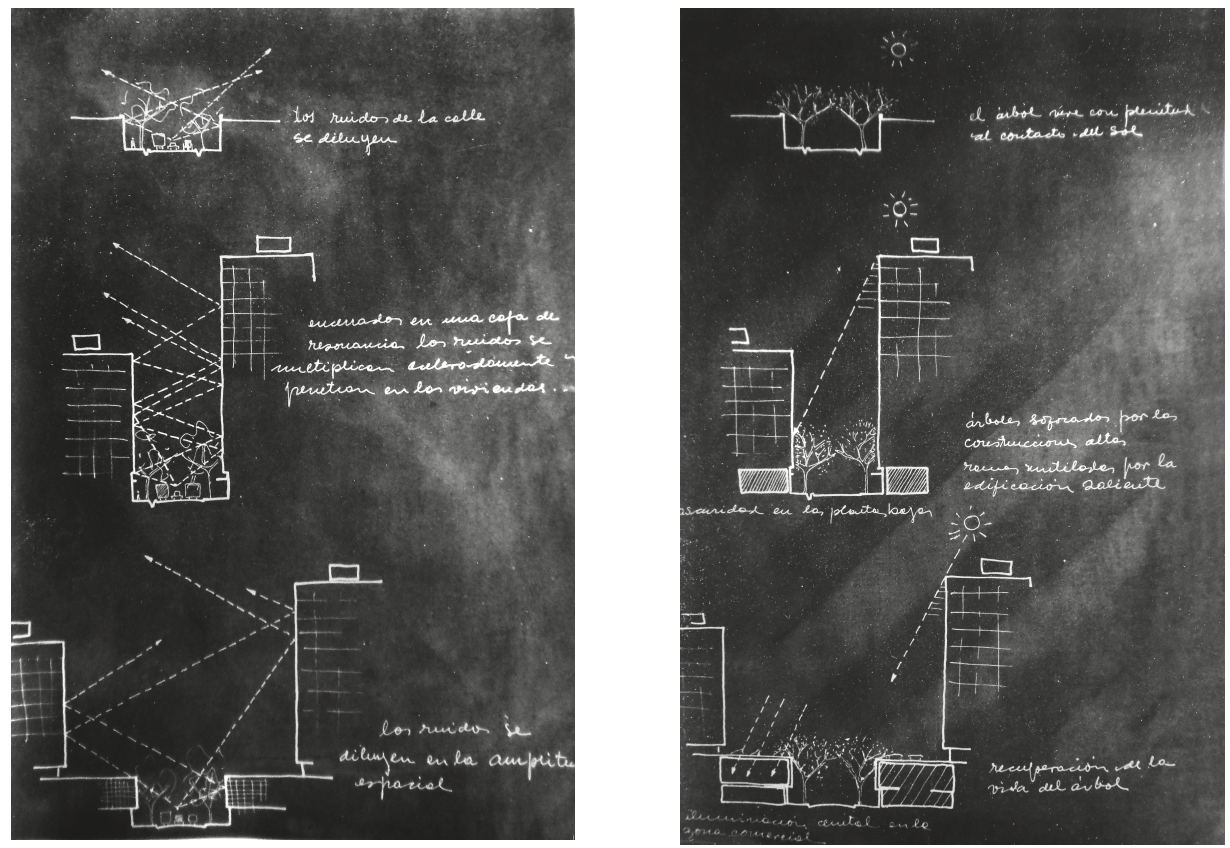

27. Sobre la disposición detallada: Municipalidad de la ciudad de Mendoza (1970). 


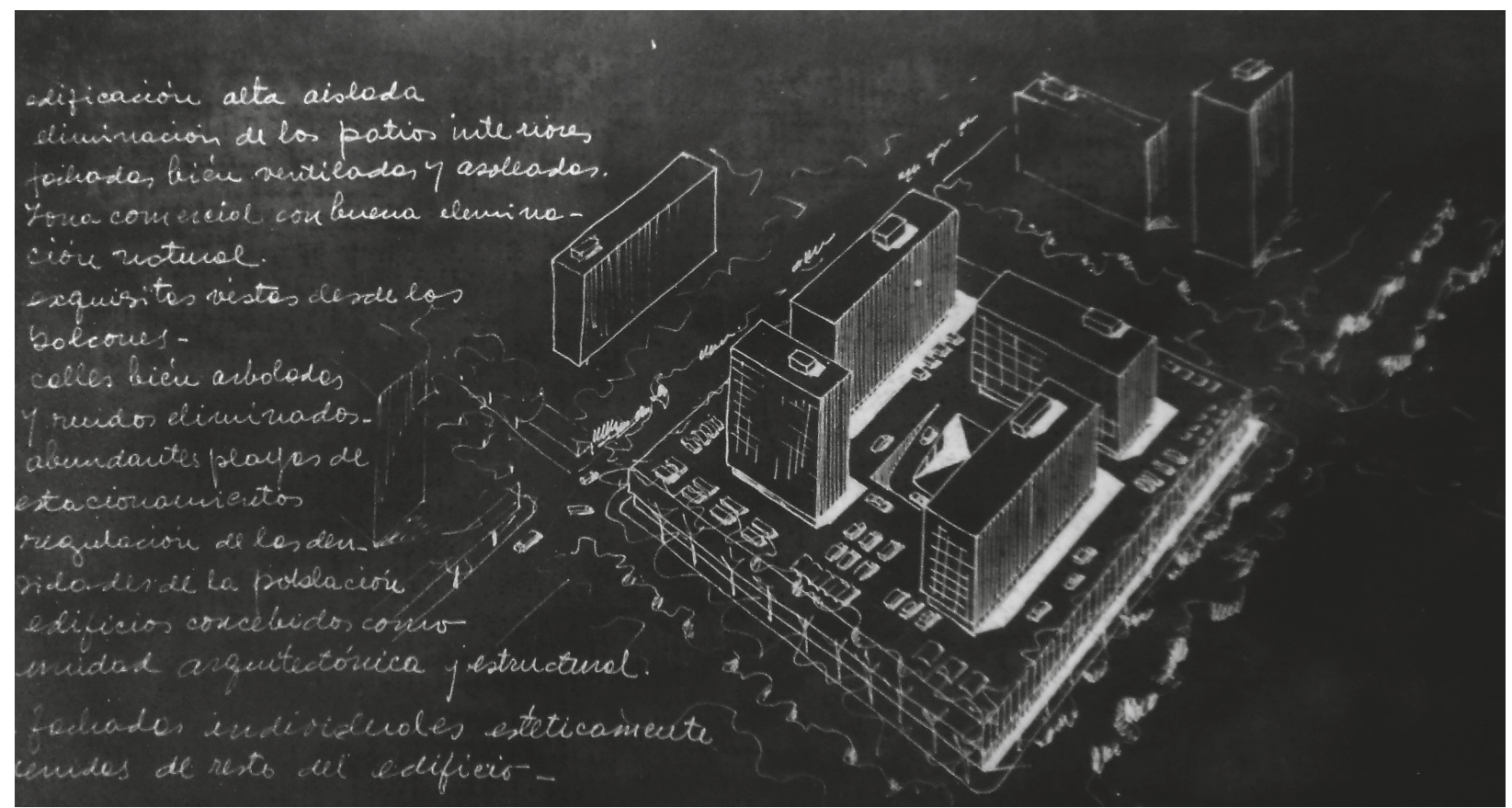

Figura 6. Propuesta de la CEPYCE sobre retiros. Fuente: Oficina de Planeamiento (1961)
Respecto de la densificación de la urbe, TEDEschr y el equipo que lo secundaba propusieron prohibir la instalación de nuevos barrios fuera de los límites de la ciudad (zona urbanizada con servicios) y fomentar la edificación donde no la hubiera y la urbanización en todas las zonas posibles dentro de la jurisdicción de la ciudad. También sugerían imponer un impuesto progresivo sobre los baldíos para incentivar la desaparición de esos vacíos urbanos; establecer una zonificación de la ciudad con la fijación de alturas mínimas y la obligatoriedad para las nuevas edificaciones de disponer de estacionamiento propio para vehículos, de acuerdo con su densidad.

Políticamente no se llegaron a implementar las limitaciones sugeridas por TEDESCHI en torno a la edificación futura en la jurisdicción de la ciudad capital. La traducción en el código del 
Teoría para la construcción de una ciudad:

Enrico Tedeschi y su vínculo con la morfología urbano-edilicia de Mendoza (Argentina, 1960)

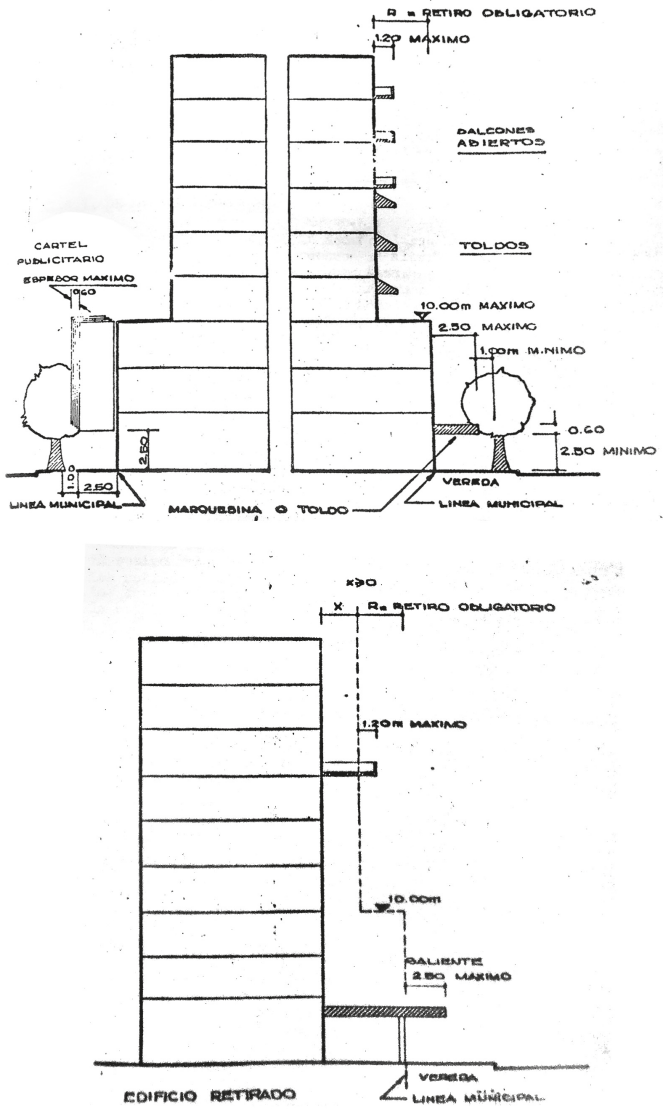

Figura 7. Retiros obligatorios del Código de edificación de 1970. Fuente: Código de edificación (1970) resto de las indicaciones fue la inclusión de un plano de zonificación con el establecimiento de los usos permitidos en cada zona y la obligatoriedad de incorporar estacionamiento vehicular en toda obra que construir. Finalmente, el código insta a en su capítulo "Normas de Carácter Urbanístico" a no alterar desfavorablemente con instalaciones varias en la vía pública el paisaje urbano o natural perjudicando las visuales.



Figura 8. Reglamentación sobre alturas mínimas en el centro de la ciudad y disposiciones sobre estacionamiento. Fuente: Oficina de Planeamiento (1961) 


\section{Primeras aproximaciones}

Si bien el informe final suponía un avance eminentemente teórico sobre la planificación de la ciudad, un "estado de la cuestión" en términos del conocimiento de la urbe, sus paisajes, demografía, actividades, etc., para lograr una planificación técnica, fue el informe previo el que representó un avance en propuestas concretas sobre el paisaje urbano de Mendoza. Los fundamentos de algunas de esas ideas fueron tomados como base para el desarrollo de la morfología urbana de la ciudad: el perfil escalonado de los edificios de gran parte de la provincia tiene su origen en aquella aproximación que el destacado urbanista y su equipo formularon para conservar el ambiente urbano mendocino.

La CEPyCE siguió existiendo luego del informe final presentado a fines de 1961, pero sin TEDEschi, Ramos Correas ni Panelo Gelly como integrantes. Los tres se embarcaron en el proyecto de la creación de la primera Facultad de Arquitectura de Mendoza, objetivo del cual participó también Simón Lacerna.

El mito de la gran influencia del informe TEDEschi sobre el código se reduce a un puñado de medidas técnicas sobre el perfil urbano de Mendoza; sin embargo, cincuenta años después, la propuesta de densificación de la ciudad de TEDEschi de alguna manera es retomada por la actual Agencia Provincial de Ordenamiento Territorial. Esta sugiere la re-densificación del Área Metropolitana de Mendoza a través de la edificación en los espacios vacíos de la mancha urbana, en lugar de urbanizar áreas irrigadas claves para sostener la matriz productiva de la provincia, aquella que TEDEschi reconocía también como fundamental en su informe final.

\section{Bibliografía y fuentes}

ADAGIO, Noemí et ál. (2017). "La valija de Enrico Tedeschi. De la posguerra italiana a la Universidad de Tucumán”. En VII Encuentro de Docentes e Investigadores en Historia del Diseño, la Arquitectura y la Ciudad. Actas. Disponible en https://rephip.unr.edu.ar/handle/2133/6794.

ALVITE, Silvia (2013). "Naturaleza crítica del espacio doméstico en la arquitectura de Enrico Tedeschi”. En Jornada de investigadores en formación, Buenos Aires: FADU-UBA, pp. 476-484. 
ALVITE, Silvia (2015), "Enrico Tedeschi y la “crítica fotográfica” en el paisaje arquitectónico latinoamericano”. En Anales del IAA, 45 (1), pp. 73-86.

BALLENT, Anahí (2009) Las huellas de la política: vivienda, ciudad, peronismo en Buenos Aires, 1943-1955, UNQ, Buenos Aires.

BÓRMIDA, Eliana (1984). “Mendoza, una ciudad oasis”. En Revista de la UM. Disponible en: http://www.um.edu.ar/ojs-new/index.php/RUM/article/view/189.

CIRVINI, Silvia y RAFFA, Cecilia (2014). "Redes, vínculos y trayectorias. Ejemplos de la progresiva autonomía del campo disciplinar y la profesión en Mendoza (1950-1970)”. En Área 20, pp. 86-101.

DIVISIÓN MENDOZA DE LA SOCIEDAD CENTRAL DE ARQUITECTOS (1953-1959) Libro de Actas.

HEALY, Mark (2012) El peronismo entre las ruinas. El terremoto y la reconstrucción de San Juan, Siglo XXI, Buenos Aires.

LIERNUR, Francisco y PSCHEPIURCA, Pablo (2012) La red austral. Obras y proyectos de Le Corbusier y sus discípulos en Argentina (1924-1965), UNQ, Buenos Aires.

MALECKI, Sebastián (2013). "Historia y crítica. Enrico Tedeschi en la renovación de la cultura arquitectónica argentina, 1950-1970”. En Ladem Utraque Europa, año 9, N. 14, pp. 137-174.

MUNICIPALIDAD DE LA CIUDAD DE MENDOZA (1970) Código de edificación de la Ciudad de Mendoza (ordenanzas 23/8850 y 21/8847). Ed. Oficial, Mendoza.

NOVICK, Alicia y PICCIONI, Raúl (2004). "Urbanismo”. En Liernur, Francisco y Aliata, Fernando (dirs.), Diccionario de Arquitectura. Tomo s | z. Clarín, Buenos Aires, pp. 134-136.

OFICINA DE PLANEAMIENTO (1961) Informe previo, mimeo.

PANELO GELLY, Raúl (1961) Reseña de la creación y labor de la Comisión Especial de Planeamiento Urbano y Código de Edificación de la ciudad de Mendoza, D’Acurzzio, Mendoza.

RAFFA, Cecilia (2014). "El plan regulador entre la técnica y la política (Mendoza 19401941)”, Bitácora 24, N. 2, pp. 31-41.

RAFFA, Cecilia (2015). "Agentes y prácticas. Biografía colectiva de la Sociedad de Arquitectos de Mendoza en sus primeros años (1953-1969)”. En Registros, año 11, N. ${ }^{0} 12$, pp. 25-40.

RAFFA, Cecilia (dir.) (2017) Arquitectos en Mendoza. Biografías, trayectorias profesionales y obras (1900-1960), IHA-FFyL-UNCuyo, Mendoza. Disponible en: http://bdigital. uncu.edu.ar/9327.

RIGOTTI, Ana María (2009). "De la ciudad al territorio, del municipio a la nación: las promesas del urbanismo como alternativa tecnocrática de gestión (1928/1958)”. En Plotkin, 
Mariano y Zimmermann, Eduardo. (comp.), Los saberes del Estado. Edhasa, Buenos Aires, pp.159-184.

RIGOTTI, Ana María (2014) Las invenciones del urbanismo en Argentina (1900-1960). Inestabiliad de sus representaciones científicas y dificultades para su profesionalización, Rosario, UNR Editora.

TEDESCHI, Enrico (1952). “Arquitectura Orgánica” (primera parte). En Nuestra Arquitectura 272, pp. 72-81.

TEDESCHI, Enrico (1954). "La enseñanza del urbanismo en las Universidades Argentinas”. En Nuestra Arquitectura 295, pp. 50-55.

TEDESCHI, Enrico (1962) Teoría de la Arquitectura, Nueva Visión, Buenos Aires.

TEDESCHI, Enrico. Legajo FAU-UM.

SELLA, Alejandra y ADAGIO, Noemí (eds.) (2013) Enrico Tedeschi. Work in progress, Idearium, Mendoza. 